\title{
Growth hormone and prolactin-staining tumors causing acromegaly: a retrospective review of clinical presentations and surgical outcomes
}

\author{
*Jonathan Rick, BS, ${ }^{1}$ Arman Jahangiri, BS, ${ }^{1}$ Patrick M. Flanigan, BS, ${ }^{2}$ Ankush Chandra, MS, ${ }^{3}$ \\ Sandeep Kunwar, MD, ${ }^{1}$ Lewis Blevins, MD, ${ }^{1}$ and Manish K. Aghi, MD, PhD'1
}

1Department of Neurosurgery, University of California, San Francisco, California; ${ }^{2}$ Cleveland Clinic Lerner College of Medicine, Cleveland, Ohio; and ${ }^{3}$ Wayne State University School of Medicine, Detroit, Michigan

\begin{abstract}
OBJECTIVE Acromegaly results in disfiguring growth and numerous medical complications. This disease is typically caused by growth hormone $(\mathrm{GH})$-secreting pituitary adenomas, which are treated first by resection, followed by radiation and/or medical therapy if needed. A subset of acromegalics have dual-staining pituitary adenomas (DSPAs), which stain for $\mathrm{GH}$ and prolactin. Presentations and treatment outcomes for acromegalics with DSPAs are not well understood.
\end{abstract}

METHODS The authors retrospectively reviewed the records of more than 5 years of pituitary adenomas resected at their institution. Data were collected on variables related to clinical presentation, tumor pathology, radiological size, and disease recurrence. The Fisher's exact test, ANOVA, Student t-test, chi-square test, and Cox proportional hazards and multiple logistic regression were used to measure statistical significance.

RESULTS Of 593 patients with pituitary adenoma, 91 presented with acromegaly. Of these 91 patients, $69(76 \%)$ had tumors that stained for GH only (single-staining somatotrophic adenomas [SSAs]), while $22(24 \%)$ had tumors that stained for GH and prolactin (DSPAs). Patients with DSPAs were more likely to present with decreased libido $(p=0.012)$, signs of acromegalic growth $(p=0.0001)$, hyperhidrosis $(p=0.0001)$, and headaches $(p=0.043)$ than patients with SSAs. DSPAs presented with significantly higher serum prolactin (60.7 vs $10.0 \mu \mathrm{g} / \mathrm{L}, p=0.0002$ ) and insulin-like growth factor-1 (IGF-1) (803.6 vs $480.0 \mathrm{ng} / \mathrm{ml}, p=0.0001)$, and were more likely to have IGF-1 levels > $650 \mathrm{ng} / \mathrm{ml}(\mathrm{n}=13$ [81.3\%] vs $n=6[21.4 \%], p=0.0001)$ than patients with SSAs despite similar sizes $(1.8$ vs $1.7 \mathrm{~cm}, p=0.5)$. Patients with DSPAs under 35 years of age were more likely to have a recurrence $(n=4[50.0 \%]$ vs $n=3[11.1 \%], p=0.01)$ than patients with SSAs under the age of 35. DSPA patients were less likely to achieve remission with surgery than SSA patients $(n=2$ $[20 \%]$ vs $n=19[68 \%], p=0.01)$. Univariate analysis identified single-staining tumors $(p=0.02)$, gross-total resection ( $p$ $=0.02$ ), and tumor diameter $(p=0.05)$ as predictors of surgical remission. Multiple logistic regression demonstrated that SSAs $(p=0.04)$ were independently associated with surgical remission of acromegaly. Kaplan-Meier analysis revealed that DSPAs had more time until disease remission $(p=0.033)$.

CONCLUSIONS Acromegalics with tumors that stain for prolactin and GH, which represented almost a quarter of acromegalics in this cohort, had more aggressive clinical presentations and postoperative outcomes than SSAs. Prolactin staining provides useful information for acromegalics undergoing pituitary surgery.

https://thejns.org/doi/abs/10.3171/2018.4.JNS18230

KEYWORDS adenoma; pituitary surgery; acromegaly; growth hormone; prolactin; outcomes

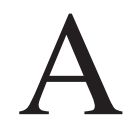
CROMEGALY, a disease characterized by excess growth hormone $(\mathrm{GH})$ that typically stems from a somatotrophic pituitary adenoma, is often recognized by the swelling of the soft tissues and bony expansion of the skull. ${ }^{4}$ These changes can cause iconic disfiguration such as frontal bossing, prognathism, macroglossia, and skin thickening. The pathophysiology of this disease also predisposes patients to numerous medical comorbidities, including cardiomegaly, rheumatism, and diabetes. ${ }^{4,6}$ However, patient presentations are markedly varied and acromegaly can manifest in many different ways. Surgery is widely regarded as the first-line therapy for acromegaly,

ABBREVIATIONS ACTH = adrenocorticotropic hormone; DSPA = dual-staining pituitary adenoma; $G H=$ growth hormone; IGF-1 = insulin-like growth factor-1; OR = odds ratio; SSA = single-staining somatotrophic adenoma.

SUBMITTED January 26, 2018. ACCEPTED April 5, 2018.

INCLUDE WHEN CITING Published online September 14, 2018; DOI: 10.3171/2018.4.JNS18230.

${ }^{*}$ J.R. and A.J. contributed equally to this work. 
with the transsphenoidal approach being suitable for the vast majority of $\mathrm{GH}$-secreting adenomas.,10,14 Reports of patients experiencing long-term remission with surgical monotherapy vary from $35 \%$ to $74 \%$, in part due to variable definitions of disease remission. ${ }^{1}$ Patients that have persistent disease are typically managed medically with somatotropin analogs, dopamine agonists, or GH antagonists, with radiosurgery or radiation therapy also playing a role in some cases. ${ }^{10,17}$ Patients who achieve biochemical remission must be followed for potential recurrence.

While most somatotrophic adenomas are monohormonal, it is estimated that up to a quarter of somatotrophic adenomas will also stain positive for prolactin.7,12,16 There are many causes for this double staining, which include dimorphous adenomas, composed of a mixture of somatotrophic and mammotrophic cells; mammosomatotrophs, tumors that originate from a common progenitor of the somatotrophs and mammotrophs; and acidophil stem cell tumors, a primitive, rare, and aggressive neoplasm. ${ }^{2,3}$ Although the etiology of dual-staining tumors has been established, the clinical impact of these tumors is poorly understood.

In this paper we address this knowledge gap by describing the clinical presentations of acromegalics with dual-staining pituitary adenomas (DSPAs) and compare them to the clinical profiles of GH single-staining somatotrophic adenomas (SSAs). We report the initial symptoms, biochemical profiles, and surgical outcomes of patients with these tumors.

\section{Methods \\ Study Cohort}

We performed a retrospective chart review of 593 surgeries for pituitary adenomas at a dedicated pituitary center. Our inclusion criteria were 1) sufficient medical records, including one comprehensive preoperative note, well-documented hospital course, and at least 1 postdischarge outpatient note; 2) unambiguous radiological imaging that could determine tumor size and location; and 3) pathological documentation, to include immunohistochemical stains confirming the presence of 1 tumor with uniform hormonal staining characteristics, comments on cellular architecture, and tumor grade. Patients were excluded if they 1) had at least one operation for pituitary adenoma before presenting to this institution; 2) had pathologic features that precluded reliable assessment of immunostaining, such as predominant cystic morphology, pituitary hemorrhage, or tumor necrosis; 3 ) had atypical cellular architecture that may represent an acidophil stem cell tumor; or 4) stained for adrenocorticotropic hormone (ACTH), as tumors that stained for all three hormones could represent a unique lineage of cells. This study was approved by the IRB at the University of California, San Francisco. Patient consent was not needed for this study, which was a retrospective chart review that used anonymized patient data.

\section{Clinical Variables}

Per our inclusion and exclusion criteria, all patients had preoperative documentation that included a compre- hensive presurgical examination (history and physical). The variables assessed were libido decrease, amenorrhea, galactorrhea, acromegalic growth (meaning the phenotypical markings of acromegaly, such as acromegalic facies, growth of digits, and enlargement of the skull and feet), hyperhidrosis, weight gain, headache, nausea, visual symptoms (meaning diplopia or vision loss), and fatigue.

\section{Pathology and Laboratory Analysis}

All samples were submitted to the Department of Pathology at the authors' institution. Specimens were subject to gross examination and subsequent immunohistochemical staining. The following features were highlighted: gross description; extent of local tumor invasion; cellular atypia; and staining for human $\mathrm{GH}$, prolactin, $\mathrm{ACTH}$, and Ki-67. Serum laboratories were also processed at the authors' institution. Reference ranges for prolactin and insulin-like growth factor (IGF) vary by patient status. For men, prolactin is taken to be normal when it is within the range of 2.1-17.7 $\mu \mathrm{g} / \mathrm{L}$. Premenopausal, nonpregnant women have a reference prolactin range of $2.8-29.2 \mu \mathrm{g} / \mathrm{L}$, pregnant women have a reference prolactin range of 9.7$208.5 \mu \mathrm{g} / \mathrm{L}$, and postmenopausal women have a prolactin reference range of $1.8-20.3 \mu \mathrm{g} / \mathrm{L}$. IGF-1 varies based on age: $108-548 \mathrm{ng} / \mathrm{ml}$ for patients $18-19.9$ years old, $83-456$ $\mathrm{ng} / \mathrm{ml}$ for patients $20-24.9$ years old, $63-373 \mathrm{ng} / \mathrm{ml}$ for patients $25-29.9$ years old, $53-331 \mathrm{ng} / \mathrm{ml}$ for patients 30 39.9 years old, $52-328 \mathrm{ng} / \mathrm{ml}$ for patients $40-49.9$ years old, 50-317 ng/ml for patients 50-59.9 years old, 41-279 $\mathrm{ng} / \mathrm{ml}$ for patients $60-69.9$ years old, 34-245 $\mathrm{ng} / \mathrm{ml}$ for patients $70-79.9$ years old, and $34-246 \mathrm{ng} / \mathrm{ml}$ for patients older than 80 years.

\section{Statistical Analysis}

All statistical analyses were performed with the JMP statistical program (version 13.0, SAS Institute Inc.). For comparisons with more than two variables with continuous dependent variables, ANOVA was used. Chi-square testing was used to compare studies with more than two proportions. Parametric comparison of two variables was conducted with the Student t-test. To compare two proportions, the Fisher's exact test was used. Kaplan-Meier analysis was used to plot time until disease remission, and significance was calculated with the Cox proportional hazards method.

\section{Results}

\section{Clinical Presentation}

\section{Demographics}

Over a 5-year period our institution performed 91 surgeries for pituitary adenomas to treat acromegaly. The 91 patients were divided based on whether their pituitary adenomas stained for GH only (SSA) or both prolactin and GH (DSPA, Fig. 1). Twenty-two patients (24.2\%) had tumors that stained for both hormones, and $69(75.8 \%)$ had tumors that only stained for $\mathrm{GH}$. The two populations had a comparable sex breakdown $(\mathrm{n}=8$ [36.4\%] women vs $\mathrm{n}=$ 32 [46.4\%] women, $\mathrm{p}=0.4)$ and mean age $(41 \pm 3.5$ years old vs $45 \pm 1.9$ years old, $\mathrm{p}=0.3$; Table 1 ). The median 
Rick et al.

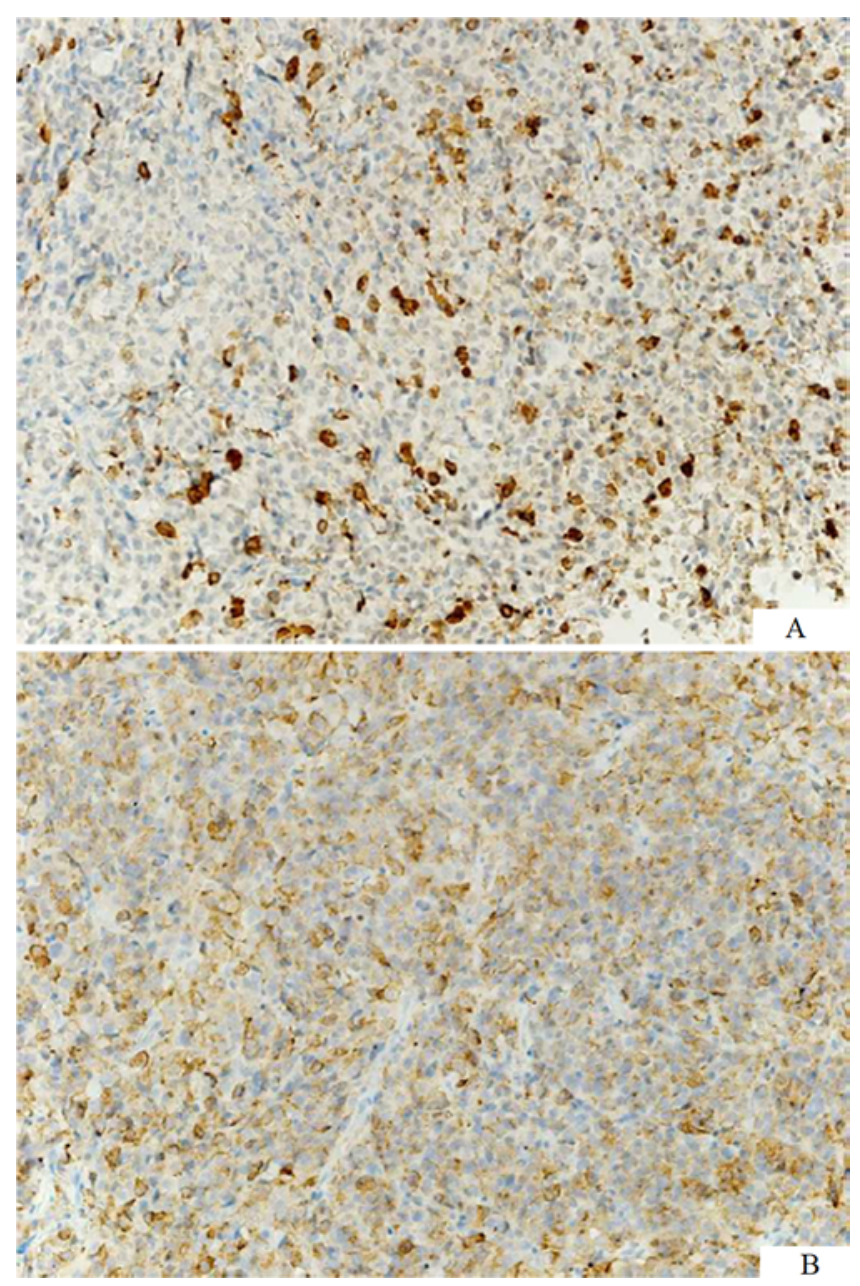

FIG. 1. Representative immunostaining of a DSPA. Shown is an example of a DSPA that exhibited robust immunostaining for human $\mathrm{GH}(\mathrm{A})$ and prolactin $(\mathrm{B})$. Bar $=80 \mu \mathrm{m}$. Figure is available in color online only.

age for dual-staining tumors was 38.4 years and for singlestaining tumors was 41.1 years.

\section{Clinical and Biochemical Profile}

Patients with dual-staining tumors were significantly more likely than patients with single-staining tumors to present with the most common symptoms of acromegaly: decreased libido (18.2\% vs $2.9 \%, \mathrm{p}=0.01)$, acromegalic features $(95.5 \%$ vs $43.5 \%, \mathrm{p}=0.0001)$, hyperhidrosis $(54.6 \%$ vs $23.2 \%, \mathrm{p}=0.0001)$, and headache $(59.1 \%$ vs $34.8 \%, \mathrm{p}=$ $0.043)$. There was no significant variation in presentations of weight gain, nausea, headache, vision changes, or fatigue between the two groups. The dual-staining patients were significantly more likely to have symptoms of galactorrhea $(22.7 \%$ vs $2.9 \%, \mathrm{p}=0.005)$. There was no significant difference in women complaining of amenorrhea between the two groups $(37.5 \%$ vs $12.5 \%, \mathrm{p}=0.09)$. In patients with DSPA, $36.4 \%$ had symptoms that could be attributed to the prolactin axis (amenorrhea, galactorrhea, or libido decrease), compared to $20.6 \%(\mathrm{p}=0.19)$ in patients with SSAs. Patients with DSPAs had a significantly higher
TABLE 1. Clinical presentation

\begin{tabular}{|c|c|c|c|}
\hline Variable & $\begin{array}{l}\text { Single- } \\
\text { Staining } \\
(n=69)\end{array}$ & $\begin{array}{l}\text { Dual- } \\
\text { Staining } \\
(n=22)\end{array}$ & $\begin{array}{c}p \\
\text { Value }\end{array}$ \\
\hline \multicolumn{4}{|l|}{ Demographics } \\
\hline Females, $\mathrm{n}(\%)$ & $32(46.4)$ & $8(36.4)$ & 0.41 \\
\hline Mean age, yrs (SD) & $45.0(1.9)$ & $40.9(3.5)$ & 0.31 \\
\hline \multicolumn{4}{|l|}{ Clinical profile, $n(\%)$} \\
\hline Libido decrease & $2(2.9)$ & $4(18.2)$ & 0.012 \\
\hline Acromegalic features & $30(43.5)$ & $21(95.5)$ & 0.0001 \\
\hline Hyperhidrosis & $16(23.2)$ & $12(54.6)$ & 0.0001 \\
\hline Weight gain & $4(5.8)$ & $1(4.5)$ & 0.822 \\
\hline Headache & $24(34.8)$ & $13(59.1)$ & 0.043 \\
\hline Nausea & $3(4.35)$ & $0(0.0)$ & 0.32 \\
\hline Vision changes & $11(15.9)$ & $2(9.1)$ & 0.42 \\
\hline Fatigue & $5(7.3)$ & $1(4.6)$ & 0.6 \\
\hline \multicolumn{4}{|l|}{ Biochemical profile } \\
\hline $\begin{array}{l}\text { Mean prolactin level, } \mu \mathrm{g} / \mathrm{L} \\
\text { (SD) }\end{array}$ & $10.0(6.7)$ & $60.7(10.5)$ & 0.0002 \\
\hline Prolactin elevated, $\mathrm{n}(\%)$ & $3 / 27(11.1)$ & $8 / 11(72.7)$ & 0.0001 \\
\hline Mean IGF-1 level, ng/ml (SD) & $480.0(41.5)$ & $803.6(54.9)$ & 0.0001 \\
\hline IGF-1 level elevated, $n(\%)$ & $69(100.0)$ & $22(100.0)$ & - \\
\hline IGF-1 >650 ng/ml, n (\%) & $6 / 28(21.4)$ & $13 / 16(81.3)$ & 0.0001 \\
\hline \multicolumn{4}{|l|}{ Tumor characteristics } \\
\hline Mean diameter, cm (SD) & $1.7(0.11)$ & $1.8(0.21)$ & 0.49 \\
\hline $\begin{array}{l}\text { Cavernous sinus invasion, } \\
\mathrm{n}(\%)\end{array}$ & $24 / 65(36.9)$ & $7(31.8)$ & 0.67 \\
\hline Mean \% Ki-67 positive (SD) & $4.3(1.1)$ & $3.3(1.6)$ & 0.62 \\
\hline
\end{tabular}

Boldface type indicates statistical significance.

mean prolactin level than patients with single-staining tumors $(60.7 \pm 10.5 \mu \mathrm{g} / \mathrm{L}$ vs $10.0 \pm 6.7 \mu \mathrm{g} / \mathrm{L}, \mathrm{p}=0.002)$. Eight $(72.7 \%)$ of the dual-staining tumors presented with elevated prolactin compared with $3(11.1 \%)$ single-staining tumors $(\mathrm{p}=0.0001)$. When single-staining tumors had elevations in prolactin they tended to be mild, ranging from 23 to $36 \mu \mathrm{g} / \mathrm{L}$ (6-16 $\mu \mathrm{g} / \mathrm{L}$ above the reference range), while prolactin elevations in dual-staining tumors ranged from 27 to $194 \mu \mathrm{g} / \mathrm{L}(10-172 \mu \mathrm{g} / \mathrm{L}$ above the reference range). While all patients with dual- or single-staining adenomas had elevated IGF-1, dual-staining tumors were associated with a significantly higher mean IGF-1 level than single-staining tumors $(803.6 \pm 54.9 \mathrm{ng} / \mathrm{ml}$ vs $480.0 \pm 41.5$ $\mathrm{ng} / \mathrm{ml}, \mathrm{p}=0.0001)$. Patients with DSPAs also were significantly more likely to have preoperative IGF-1 levels above $650 \mathrm{ng} / \mathrm{ml}(\mathrm{n}=13$ [81.3\%] vs $\mathrm{n}=6$ [21.4\%], $\mathrm{p}=0.0001)$, a threshold defined by recursive partitioning. Lastly, DSPAs that did not present with elevated prolactin on serology still had significantly higher IGF-1 preoperatively (734.0 vs $480.0 \mathrm{ng} / \mathrm{ml}, \mathrm{p}=0.003$ ). Despite the greater degree of IGF-1 elevation found in patients with DSPAs, the two cohorts had similar tumor radiological appearances, with dual- and single-staining tumors having mean diameters of 1.8 and $1.7 \mathrm{~cm}$, respectively $(\mathrm{p}=0.49)$, and single- and dual-staining tumors having similar rates of cavernous si- 
TABLE 2. Patient outcomes

\begin{tabular}{lccc}
\hline \multicolumn{1}{c}{ Variable } & Single-Staining & Dual-Staining & $\mathrm{p} \mathrm{Value}$ \\
\hline Surgical profile, $\mathrm{n}(\%)$ & & & \\
\hline Subtotal resection & $21 / 58(36.2)$ & $7 / 21(33.3)$ & 0.81 \\
\hline CSF leak & $8(11.6)$ & $4(18.2)$ & 0.4 \\
\hline Lumbar drain & $16(23.2)$ & $8(36.4)$ & 0.22 \\
\hline Mean follow-up, yrs (SD) & $3.5(0.4)$ & $3.4(0.7)$ & 0.88 \\
\hline Recurrence, $\mathrm{n}(\%)$ & & & \\
\hline Overall recurrence & $5(7.2)$ & $4(18.2)$ & 0.07 \\
\hline Recurrence w/in 1 yr & $2(40.0)$ & $4(100.0)$ & 0.11 \\
\hline Age $>35$ yrs at diagnosis & $2 / 42(4.8)$ & $0 / 14(0.0)$ & 0.5 \\
\hline Age $<35$ yrs at diagnosis & $3 / 27(11.1)$ & $4 / 8(50.0)$ & 0.01 \\
\hline Boldace type idicas
\end{tabular}

Boldface type indicates statistical significance.

nus invasion ( $\mathrm{p}=0.67$; Table 1$)$. Single- and dual-staining tumors had comparable staining for Ki-67, with a mean of $4.3 \% \pm 1.1 \%$ of single-staining adenoma cells staining positive compared with $3.3 \% \pm 1.6 \%$ of dual-staining adenoma cells $(\mathrm{p}=0.62)$.

\section{Patient Postoperative Outcomes}

Postoperatively there was no significant variation in CSF leaks, need for lumbar drains, or achieved extent of resection (Table 2). Single-staining tumor patients were significantly more likely to experience postoperative biochemical remission without any medication $(\mathrm{n}=19$ [67.9\%] vs $\mathrm{n}=2$ [20.0\%], $\mathrm{p}=0.01$; Table 3). Both groups had comparable follow-up times (Table 2). In our study, dualstaining tumors recurred more frequently $(\mathrm{n}=4$ [18.2\%] vs $n=5[7.2 \%]$ ), but this finding only trended toward significance $(\mathrm{p}=0.07)$. Of note, all tumor recurrences in the dual-staining group occurred in patients under the age of 35 and ultimately $50 \%$ of patients with dual-staining tumors in this younger age group had a recurrence.

\section{Adjuvant Therapy for Patients Failing to Achieve Postoperative Biochemical Remission}

Patients who remained symptomatic postoperatively and had a radiologically identified mass were referred for radiotherapy. In total, 11 patients underwent radiotherapy: 2 (9.1\%) DSPA patients and $9(15.8 \%)$ single-staining tumor patients were treated with Gamma Knife surgery ( $p$ $=0.44$ ). Of the patients who underwent radiotherapy, none of the DSPA patients experienced disease remission after treatment and required medical management for disease remission. Four (44.4\%) of the single-staining tumor patients experienced a biochemical remission after radiosurgery and did not require additional medical management $(\mathrm{p}=0.21)$.

Of the 91 patients who were surgically treated at our institution, 38 (41.8\%) underwent additional postoperative adjuvant medical treatment managed at our institution and thus had comprehensive records and documented follow-up past 1 year. Twenty-eight (40.5\%) of the original 69 patients with single-staining tumors and $10(45.5 \%)$ of the original 22 patients with dual-staining tumors had
TABLE 3. Disease remission

\begin{tabular}{lccc}
\hline \multicolumn{1}{c}{ Variable } & $\begin{array}{c}\text { Single-Staining } \\
(\mathrm{n}=28)\end{array}$ & $\begin{array}{c}\text { Dual-Staining } \\
(\mathrm{n}=10)\end{array}$ & $\begin{array}{c}\mathrm{p} \\
\text { Value }\end{array}$ \\
\hline Medication for remission & & & \\
\hline No medication & $19(67.9)$ & $2(20.0)$ & 0.01 \\
\hline Any medication & $9(32.1)$ & $8(80.0)$ & \\
\hline No. of medications & & & \\
\hline 1 & $9(32.1)$ & $5(50.0)$ & - \\
\hline 2 & $0(0.0)$ & $2(20.0)$ & \\
\hline 3 & $0(0.0)$ & $1(10.0)$ & \\
\hline $\begin{array}{c}\text { Mean medication dosages, } \\
\text { mg (SD) }\end{array}$ & & & \\
\hline Pegvisomant & $5.65(2.3)$ & $16.4(4.1)$ & 0.03 \\
\hline Cabergoline* & $0(0.17)$ & $0.9(0.33)$ & 0.02 \\
\hline Lanreotide† & $4.7(6.9)$ & $52.5(15.1)$ & 0.009 \\
\hline $\begin{array}{c}\text { Patients on max dosage, } \\
\mathrm{n}(\%)\end{array}$ & & & \\
\hline Pegvisomant & $1(3.6)$ & $3(30.0)$ & 0.04 \\
\hline $\begin{array}{c}\text { Cabergoline* } \\
\text { Lanreotide† }\end{array}$ & $0(0.0)$ & $0(0.0)$ & - \\
\hline
\end{tabular}

Boldface type indicates statistical significance.

${ }^{*}$ Administered twice a week.

† Administered every 4 weeks.

long-term medical follow-up at our institution (Table 3). Eight DSPA patients required medical therapy to achieve remission compared with 9 single-staining tumors. Four (50.0\%) of the DSPA patients required medical therapy despite gross-total resection versus $3(33.3 \%)$ of the singlestaining tumor patients. All patients with single-staining tumors that required medication to achieve remission were able to do so with 1 agent. For dual-staining tumor patients, 5 needed only 1 medication, 2 needed 2 medications (pegvisomant and lanreotide), and 1 required 3 medications (pegvisomant, cabergoline, and lanreotide). The mean postoperative doses of pegvisomant (16.4 vs $5.63 \mathrm{mg}$ daily, $\mathrm{p}=0.03$ ), cabergoline (0.9 vs $0.0 \mathrm{mg}$ biweekly, $\mathrm{p}=$ 0.02 ), and lanreotide (52.5 vs $4.7 \mathrm{mg}$ monthly, $\mathrm{p}=0.009$ ) that were needed were significantly higher in the dualstaining group. The dual-staining group had more patients on the maximum dosage of pegvisomant $(\mathrm{n}=3[30.0 \%]$ vs $\mathrm{n}=1[3.6 \%], \mathrm{p}=0.04)$ and lanreotide $(\mathrm{n}=2[20.0 \%]$ vs $\mathrm{n}$ $=0[0.0 \%], \mathrm{p}=0.03$ ).

We next analyzed the time until patients achieved longterm biochemical remission, as defined by IGF-1 levels persistently below acromegalic levels. The median time for disease remission for single-staining tumors was 2.9 months vs 13.8 months for DPSAs (Fig. $2, \mathrm{p}=0.03$ ). The $3-, 6-, 12-$, and 24-month remission rate was $51.6 \%, 58.9 \%$, $71.7 \%$, and $93.2 \%$ for single-staining tumors and $44.7 \%$, $44.7 \%, 44.7 \%$, and $59.9 \%$ for DSPAs, respectively.

\section{Predictors of Long-Term Remission After Surgery}

We performed a univariate analysis on the patients with comprehensive endocrinological follow-up $(\mathrm{n}=35)$ to identify predictors of surgical remission (Table 4). 


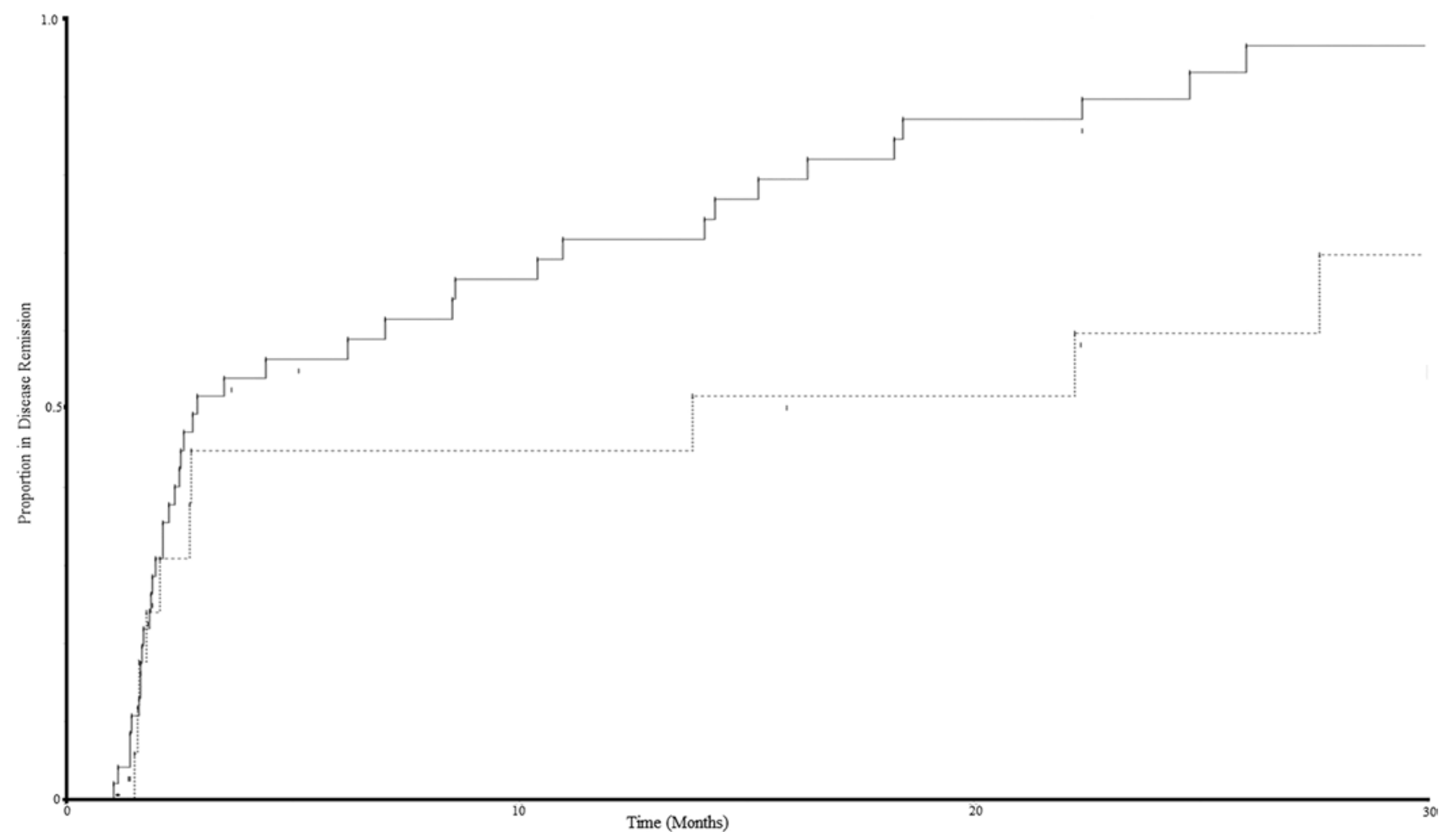

FIG. 2. Kaplan-Meier analysis of disease remission by prolactin immunohistochemistry. Time (in months) until patients achieve biochemical disease remission with single-staining tumors (solid line) compared to dual-staining tumors (dashed line; $p=0.033$ ).

Single-staining tumors (odds ratio $[\mathrm{OR}]=7.0, \mathrm{p}=0.02$ ), gross-total resection $(\mathrm{OR}=5.4, \mathrm{p}=0.02)$, and tumor diameter $(\mathrm{OR}=3.1, \mathrm{p}=0.05)$ were significantly associated with remission after surgery alone. Cavernous sinus invasion and acromegalic growth at presentation were not significantly associated with remission after surgery alone (Table 4). The significant univariate predictors were analyzed via multinomial logistic regression (Table 5). Only single-staining tumors $(\mathrm{OR}=8.6, \mathrm{p}=0.04)$ remained significantly associated in this model.

\section{Discussion}

Acromegaly is a disease that has significant heterogeneity in presentation and surgical outcomes. We analyzed the impact of prolactin immunopositivity on the presentation and postoperative outcomes of patients with acro-

TABLE 4. Univariate predictors of surgical cure

\begin{tabular}{llcl}
\hline \multicolumn{1}{c}{ Variable } & OR & $95 \% \mathrm{Cl}$ & $\mathrm{p}$ Value \\
\hline Tumor diameter & 3.1 & $1.0-9.8$ & $\mathbf{0 . 0 5}$ \\
\hline Gross-total resection & 5.4 & $1.19-24.5$ & $\mathbf{0 . 0 2}$ \\
\hline Single-staining tumor & 7 & $1.2-40.8$ & $\mathbf{0 . 0 2}$ \\
\hline Acromegalic growth & 4.33 & $0.93-20$ & 0.052 \\
\hline Cavernous sinus invasion & 2.5 & $0.6-9.7$ & 0.18 \\
\hline
\end{tabular}

$\mathrm{Cl}=$ confidence interval.

Boldface type indicates statistical significance. megaly. We identified a number of significant differences in patient presentation and clinical outcomes based on this marker, suggesting that prolactin immunostaining identifies a subset of more aggressive somatotrophic adenomas.

We found that about a quarter of our patients had dualstaining tumors, which agrees with findings from other studies. ${ }^{7,16}$ While patients with these dual-staining tumors had a similar sex breakdown and age range as the singlestaining tumors, patients with dual-staining tumors were more likely to present with many of the characteristic symptoms of acromegaly and had greater elevation of IGF1 , indicating more severe clinical and biochemical acromegaly. Not surprisingly, these patients with dual-staining adenomas also had other potentially hyperprolactinemiainduced symptoms, such as decreased libido, but the presence of these additional symptoms did not cause them to present earlier in their disease course based on our finding of comparable tumor size in dual- versus single-staining adenomas. Hyperprolactinemia was not common in SSAs, but those that did have elevations typically had values be-

TABLE 5. Multinomial logistic regression of surgical cure

\begin{tabular}{llrl}
\hline \multicolumn{1}{c}{ Variable } & OR & \multicolumn{1}{c}{$95 \% \mathrm{Cl}$} & $\mathrm{p}$ Value \\
\hline Tumor diameter & 1.09 & $0.008-183.9$ & 0.9 \\
\hline Gross-total resection & 9.6 & $0.9-141.6$ & 0.06 \\
\hline Single-staining tumor & 8.6 & $1.09-109.7$ & 0.04 \\
\hline
\end{tabular}

Boldface type indicates statistical significance. 
low $40 \mathrm{ng} / \mathrm{ml}$, which is a value typically observed secondary to stalk effect. ${ }^{5}$ For dual-staining tumors, more than half of the patients had prolactin elevations higher than the cutoff of 41.5, which, when accounting for tumor size, would favor tumor hormonal secretion over stalk effect. ${ }^{5}$ It is also notable, however, that $27.3 \%$ of dual-staining tumor patients did not have prolactin elevation, but patients with dual-staining tumors without elevated prolactin still had more severe IGF-1 elevation and symptoms than singlestaining tumor patients, indicating that the dual-staining adenomas confer a more aggressive phenotype even if their prolactin component is biochemically silent.

In addition to differences in patient presentation, patients with dual-staining tumors had some notable differences in postoperative outcomes. Even after a radiological gross-total resection, patients with acromegaly may not experience biochemical remission after surgery. ${ }^{5}$ It is difficult to predict who will ultimately experience remission and if surgery will, at minimum, reduce the patient medical burden. We found that $80 \%$ of patients with dualstaining tumors did not experience a surgical remission. This finding contrasts greatly with the $32 \%$ persistence rate of single-staining tumors. Additionally, all patients with single-staining tumors were able to achieve disease remission with a single agent, which contrasted with the $30 \%$ of patients with dual-staining tumors who required polypharmacy for disease control. Dual-staining tumor patients had higher mean dosages for pegvisomant, cabergoline, and lanreotide. Additionally, almost a third and a fifth of DSPA patients required the maximum dosage of pegvisomant and lanreotide, respectively. Ultimately, we found that DSPAs were less likely to experience remission from surgery and, for patients who had persistent disease, require more than 1 medication to control their disease.

We also sought to identify predictors of long-term remission after surgery alone. Our univariate analysis identified tumor diameter, extent of resection, and monohormonal tumors to be significantly associated with surgical remission. When these findings were extended to multinominal logistic regression, only monohormonal tumors were an independent predictor of surgical remission, underscoring the importance of prolactin staining as a predictor of surgical remission in acromegalics. An interesting correlate of these findings was how much longer patients with DSPAs suffered from elevated IGF-1 levels. We found that single-staining tumors took a median of 2.9 months until their IGF-1 levels achieved therapeutic ranges, whereas DSPAs took a median of 13.8 months. The persistence in these elevations is likely due to the fact that surgery was less likely to be curative and that these patients required more time to titrate their medications. Thus, patients with DSPAs were likely to suffer from biochemical acromegaly for significantly longer periods of time after their operation.

The impact of dual-secreting tumors has been explored by other investigators, but these studies have focused on the hormonal axes rather than adenoma staining. First among these is Wang et al., who found that elevations in two hormonal axes were associated with slightly lower surgical remission rates.$^{18}$ It is interesting that we report a similar effect, despite taking a different path to ascertain dual-hormonal status. In the absence of staining data in their analysis, the prolactin elevations in their cases could be caused by somatotrophs causing stalk effect, or dualsecreting tumors, which could have led to this team finding less robust associations. The serum hormone approach was also used by Huan et al. as they compared elevations in prolactin to elevations in prolactin and $\mathrm{GH} .{ }^{8}$ This team reported higher levels of prolactin in patients that only had monohormonal imbalance, implying that pure prolactinomas were more hormonally active than dual-secreting tumors. This team also found that double hormone elevation was associated with increased risk of recurrence. In our study we did not find that serum hormone elevations were particularly associated with postsurgical outcomes; in this way, we agree more with the work by Wang et al., as opposed to Huan et al. Instead, sorting acromegalic patients into monohormonal or dual-hormonal based on staining status had the most clinical utility. Although DSPAs had significantly higher levels of IGF-1 and prolactin, no cutoff was particularly useful in predicting DSPAs preoperatively as a good number of them had quite minor elevations.

It is also not entirely clear what the etiology of these dual-staining tumors is, but two possibilities exist. First, one study found that a full cohort of 14 dual-staining tumors were, in fact, mammosomatotrophic adenomas. ${ }^{16}$ However, mRNA analysis has also identified that most somatotrophic adenomas produce prolactin, even if it is below the threshold of typical immunohistochemistry detection. ${ }^{11}$ This could reflect a spectrum of relatively welldifferentiated somatotrophic adenomas, which express minimal prolactin, to more primitive mammosomatotrophic adenomas. A second possibility is that dual-staining tumors could reflect a mixture of mammotrophic and somatotrophic adenomas that are each exerting endocrine effects. Distinguishing between these two possibilities will require molecular analysis, or visualization via electron microscopy. ${ }^{11,15}$ Such molecular analysis could also provide insight into what caused the more aggressive clinical and biochemical behavior we found in dual-staining adenomas. Molecular analysis may also enable more precise characterization of somatotrophic pituitary adenomas than immunostaining, whose poor correlation with RNA has been described. ${ }^{9,13}$ Until these molecular studies are completed, immunostaining, which is commonly recorded in pathology reports, will have significant clinical utility, including for the reasons described in our study.

\section{Study Limitations}

As a retrospective review, our study is subject to the usual limitations of retrospective analysis. As mentioned in the introduction, dual-staining tumors can represent a few unique lineages, namely mammosomatotrophs and dimorphous adenomas. Our study is not powered or designed to separate out the profiles of these exact tumor groups. However, the standard operating procedure in most clinical pathology practices typically does not differentiate these tumor types either, which makes the dual-staining phenotype the most reliably reported finding. Therefore, the findings reported in this study are placed to have broad clinical applicability. 


\section{Future Directions}

One potential advancement in the future will be the use of gene expression analysis to provide even more sensitive assays for adenoma diagnosis than immunohistochemistry. ${ }^{914}$ Gene expression analysis may also allow identification of hematological markers that are better associated with the diagnosis than IGF-1 or prolactin. More efficient diagnosis of these patients will allow us to better identify and characterize these tumors and specifically tailor treatment to patients affected by these rare adenomas.

\section{Conclusions}

Acromegalics with tumors that stain for prolactin and $\mathrm{GH}$, which represented almost a quarter of acromegalics in our cohort, had significantly more aggressive clinical presentations, as reflected in disease symptoms and laboratory values, than patients with single-staining tumors. Patients with dual-staining tumors were less likely to experience remission from surgery alone and require additional medical management to control their disease. Therefore, prolactin staining provides useful prognostic information for acromegalics undergoing pituitary surgery.

\section{Acknowledgments}

Jonathan Rick, Arman Jahangiri, and Ankush Chandra were supported by the Howard Hughes Medical Institute.

\section{References}

1. Abu Dabrh AM, Mohammed K, Asi N, Farah WH, Wang Z, Farah $\mathrm{MH}$, et al: Surgical interventions and medical treatments in treatment-naïve patients with acromegaly: systematic review and meta-analysis. J Clin Endocrinol Metab 99:4003-4014, 2014

2. Al-Brahim NYY, Asa SL: My approach to pathology of the pituitary gland. J Clin Pathol 59:1245-1253, 2006

3. Arafah BM, Nasrallah MP: Pituitary tumors: pathophysiology, clinical manifestations and management. Endocr Relat Cancer 8:287-305, 2001

4. Chanson P, Salenave S, Kamenicky P: Acromegaly. Handb Clin Neurol 124:197-219, 2014

5. Cheng JS, Salinas R, Molinaro A, Chang EF, Kunwar S, Blevins L, et al: A predictive algorithm for evaluating elevated serum prolactin in patients with a sellar mass. J Clin Neurosci 22:155-160, 2015

6. Giustina A, Chanson P, Kleinberg D, Bronstein MD, Clemmons DR, Klibanski A, et al: Expert consensus document: A consensus on the medical treatment of acromegaly. Nat Rev Endocrinol 10:243-248, 2014

7. Halmi NS: Occurrence of both growth hormone- and prolactin-immunoreactive material in the cells of human somatotropic pituitary adenomas containing mammotropic elements. Virchows Arch A Pathol Anat Histopathol 398:19-31, 1982

8. Huan C, Cui G, Ren Z: The characteristics of acromegalic patients with hyperprolactinemia and the differences with hyperprolactinemia patients. Pak J Pharm Sci 28 (2 Suppl):713-718, 2015
9. Jiang X, Zhang X: The molecular pathogenesis of pituitary adenomas: an update. Endocrinol Metab (Seoul) 28:245254,2013

10. Katznelson L, Laws ER Jr, Melmed S, Molitch ME, Murad $\mathrm{MH}$, Utz A, et al: Acromegaly: an endocrine society clinical practice guideline. J Clin Endocrinol Metab 99:3933-3951, 2014

11. Lloyd RV, Cano M, Chandler WF, Barkan AL, Horvath E, Kovacs K: Human growth hormone and prolactin secreting pituitary adenomas analyzed by in situ hybridization. Am J Pathol 134:605-613, 1989

12. Manuylova E, Calvi LM, Hastings C, Vates GE, Johnson MD, Cave WT Jr, et al: Late presentation of acromegaly in medically controlled prolactinoma patients. Endocrinol Diabetes Metab Case Rep 2016:16-0069, 2016

13. Mao ZG, He DS, Zhou J, Yao B, Xiao WW, Chen CH, et al: Differential expression of microRNAs in GH-secreting pituitary adenomas. Diagn Pathol 5:79, 2010

14. Muhammad A, van der Lely AJ, Neggers SJCMM: Review of current and emerging treatment options in acromegaly. Neth J Med 73:362-367, 2015

15. Rahman M, Jusué-Torres I, Alkabbani A, Salvatori R, Rodríguez FJ, Quinones-Hinojosa A: Synchronous GH- and prolactin-secreting pituitary adenomas. Endocrinol Diabetes Metab Case Rep 2014:140052, 2014

16. Robert F, Pelletier G, Serri O, Hardy J: Mixed growth hormone and prolactin-secreting human pituitary adenomas: a pathologic, immunocy tochemical, ultrastructural, and immunoelectron microscopic study. Hum Pathol 19:1327-1334, 1988

17. Rowland NC, Aghi MK: Radiation treatment strategies for acromegaly. Neurosurg Focus 29(4):E12, 2010

18. Wang M, Mou C, Jiang M, Han L, Fan S, Huan C, et al: The characteristics of acromegalic patients with hyperprolactinemia and the differences in patients with merely GH-secreting adenomas: clinical analysis of 279 cases. Eur J Endocrinol 166:797-802, 2012

\section{Disclosures}

The authors report no conflict of interest concerning the materials or methods used in this study or the findings specified in this paper.

\section{Author Contributions}

Conception and design: Rick, Flanigan, Chandra. Acquisition of data: Rick, Jahangiri, Flanigan, Kunwar, Blevins. Analysis and interpretation of data: Rick. Drafting the article: Rick. Critically revising the article: Aghi, Rick, Flanigan. Reviewed submitted version of manuscript: all authors. Statistical analysis: Rick. Study supervision: Aghi.

\section{Correspondence}

Manish K. Aghi: California Center for Pituitary Disorders, University of California, San Francisco, CA. aghim@neurosurg.ucsf. edu. 